\title{
Interposition de péritoine latéro-pelvien pour éviter le risque de fistule recto-vaginale après résection colorectale avec colpotomie pour
}

\section{endométriose}

J.-D.Hini

C.Ferrier

C.Owen

A.Arfi

C.Touboul

S.Bendifallah

E.Darai

8

Service de chirurgie gynécologique, hôpital Tenon, 4, rue de la Chine, 75012 Paris, France

\section{Introduction}

11 La prévalence des localisations digestives de l'endométriose n'est pas précisément connue

12 mais estimée à 5-12\% des patientes atteintes de cette maladie [1, 2]. Dans 80 à $90 \%$ des cas,

13 ces lésions concernent le rectum et/ou la charnière recto sigmoïdienne [3]. Un traitement

14 chirurgical est requis en cas d'échec d'un traitement médical bien conduit ou en cas

15 d'infertilité avec échec de l'assistance médicale à la procréation [4]. Selon l'étude Friends

16 impliquant la plupart des centres experts français, au moins 1135 patientes ont été opérée

17 d'une endométriose colorectale en 2015 [5].

18 Les modalités du traitement chirurgical de l'endométriose colorectale peuvent être soit un

19 traitement conservateur (shaving rectal ou résection discoïde) soit un traitement radical

20 (résection segmentaire) en fonction de la localisation, du caractère multifocal, de la taille et de

21 l'extension circonférentiel de la lésion [6, 7]. L’atteinte colorectal requiert souvent des gestes

22 chirurgicaux associés, principalement l’urétérolyse, la résection du torus uterinum, des

23 ligaments utéro-sacrés, du paramètre, de la lame sacro-génito-pubienne et, dans 20\% des cas,

24 une colpotomie ou colpectomie partielle [8]. La résection concomitante vaginale et 
colorectale expose les patientes au risque de fistule recto-vaginale qui représente l'une des complications les plus sévères. Une méta-analyse récente estime l'incidence de cette complication à 2,5\% en cas de geste de résection rectale (2 à 6\% selon les études)[9, 10,11]. Le risque semble toutefois moins élevé en cas de résection discoïde [11, 12]. Afin de réduire le risque de fistules recto-vaginale, différents procédés chirurgicaux ont été décrits. Le plus citée est la stomie prophylactique, dont l'efficacité a été étudiée dans le cadre du cancer colorectal par deux méta-analyses [13, 14]. Ces deux méta-analyses montrent une diminution significative du risque de fistule anastomotique et de reprise chirurgicale en cas de stomie prophylactique après résection colorectale avec anastomose digestive basse. Pour l'endométriose, seules deux séries rétrospectives suggèrent une diminution du taux de fistule recto-vaginale chez les patientes ayant bénéficié d'une stomie de protection après résection colorectale, associée à une résection vaginale, mais sans atteindre la significativité statistique [15, 16]. Par ailleurs, la réalisation d'une stomie a un impact négative sur la qualité de vie y compris après rétablissement de la continuité $[17,18]$, et est source de complications spécifiques [19].

Des techniques d'épiplooplastie ont été suggérées comme une alternative à la stomie.

Cependant, une méta-analyse en chirurgie colorectale ne retrouvait pas de bénéfice sur le taux de fistule, de mortalité ou de reprise chirurgicale [20]. Plus récemment, pour les patientes bénéficiant d'une hystérectomie totale associée à une résection colorectale pour endométriose, une technique d'interposition de péritoine pré-vésical a été décrite [21]. Le bénéfice de cette technique a été étudié dans une petite étude de cohorte rétrospective [22]. Aucune fistule recto-vaginale n'a été observée chez les 27 patientes opérées d'une hystérectomie totale associée à une résection colorectale (transversale ou discoïde) pour endométriose avec interposition de péritoine pré-vésical. 
49 Dans cet article, nous décrivons une technique d'interposition de péritoine pelvien latéral afin

50 de réduire les risques de fistule recto-vaginale chez une patiente opérée d'une résection 51 d'endométriose colorectale sans hystérectomie associée.

52 


\section{Technique chirurgicale}

54 Nous décrivons l'intervention de Mme Z. opérée d'une endométriose symptomatique. Elle

55 présentait une endométriose profonde avec une atteinte du torus uterinum, des ligaments utéro

56 sacrés, du vagin, ainsi qu'une infiltration digestive rectale sur $21 \mathrm{~mm}$ de hauteur et sur $60^{\circ}$ de circonférence. Nous avons réalisé une cœlioscopie robot-assistée.

La patiente était installée en décubitus dorsal, bras le long du corps, en position de laparoscopie double-équipe. Une sonde urinaire à demeure et une canulation utérine ont été mis en place en début d'intervention.

L'intervention a débuté par une urétérolyse extensive gauche et l'ouverture de la fosse para rectale gauche. Les lésions du ligament utéro-sacré gauche et du torus utérinum ont été réséquées électivement. La lésion vaginale a été disséquée, à l'aide de bougies vaginale et rectale, puis réséquée électivement en réalisant un shaving vaginal. Au cours de cette procédure, une ouverture du vagin sur environ $1 \mathrm{~cm}$ est survenue. L'étape suivante consistait à réséquer la lésion digestive. Pour cela il a été nécessaire d’ouvrir les fosses para-rectales, d'effectuer un shaving rectal pour réséquer la partie exophytique de la lésion digestive et repérer la zone d’infiltration digestive. La taille et la circonférence de la lésion infiltrant la paroi rectale permettaient de réaliser une résection discoïde selon la technique déjà décrite (20). Une fois l'ensemble des lésions endométriosiques retiré (Figure 1), la zone de colpotomie a été suturée et recouverte par du péritoine. Pour cela, le péritoine pelvien latéral droit a été incisé de façon rectangulaire en dessous et en dedans de l'uretère droit. Le péritoine a été disséqué superficiellement jusqu'au croisement de l'artère utérine avec l’uretère en regard du paramètre droit. (Figure 2). La découpe péritonéale restait rattachée au péritoine pariétal sur $2 \mathrm{~cm}$ pour préserver une vascularisation (figure 3). Après rotation partielle du lambeau péritonéale, celui-ci a été suturé par 4 points de vicryl 2/0 à la face postérieure du 
vagin recouvrant ainsi la zone de colpotomie (figure 4).

78

Les suites opératoires ont été simples.

79

80

81

\section{Conclusion}

83 L’interposition du péritoine latéro-pelvien est un artifice simple permettant d'interposer un

84 lambeau de tissu sain entre la cicatrice vaginale et colorectale évitant le recours à une

85 dérivation intestinale et réduisant le risque de survenue d'une fistule recto-vaginale.

86 Cependant, cette technique ne peut être utilisée qu’à la condition d'avoir un péritoine pelvien

87 latéral sain, dépourvu de lésion d’endométriose. Par ailleurs, même s'il n'est pas étudié dans

88 cet article, le risque opératoire associé à cette technique est a priori très faible. Une

89 urétérolyse bilatérale est généralement effectuée préalablement au geste de résection

90 colorectale dans ce type de chirurgies, rendant le risque urétéral faible. De plus le décollement

91 péritonéal étant superficiel, le risque de plaie vasculaire est faible. D'autres études sont

92 nécessaires pour étudier la vitalité du lambeau après sa mise en place, ainsi que son efficacité

93 dans la réduction du risque de fistule recto-vaginale.

94

95 Déclaration des liens d'intérêt.

96 Les auteurs déclarent ne pas avoir de lien d’intérêt.

97

98 
100 1. Weed JC, Ray JE. Endometriosis of the bowel. Obstet Gynecol. mai 1987;69(5):727-30.

1012 2. Jerby BL, Kessler H, Falcone T, Milsom JW. Laparoscopic management of colorectal

9. Tarjanne S, Heikinheimo O, Mentula M, Härkki P. Complications and long-term followup on colorectal resections in the treatment of deep infiltrating endometriosis extending to bowel wall. Acta Obstet Gynecol Scand. janv 2015;94(1):72-9.

10. Balla A, Quaresima S, Subiela JD, Shalaby M, Petrella G, Sileri P. Outcomes after rectosigmoid resection for endometriosis: a systematic literature review. Int J Colorectal Dis. juill 2018;33(7):835-47.

11. Abo C, Moatassim S, Marty N, Saint Ghislain M, Huet E, Bridoux V, et al. Postoperative complications after bowel endometriosis surgery by shaving, disc excision, or segmental resection: a three-arm comparative analysis of 364 consecutive cases. Fertil Steril. 2018;109(1):172-178.e1.

12. Jayot A., Bendifallah S., Abo C., Arfi A., Owen C., Darai E. «Feasibility, Complications, and Recurrence after Discoid Resection for Colorectal Endometriosis: A Series of 93 Cases ». J Minim Invasive Gynecol [En ligne]. janvier 2020. Vol. 27, n¹, p. 212- 219. Disponible sur : < https://doi.org/10.1016/j.jmig.2019.07.011 > 
13. Belghiti J, Ballester M, Zilberman S, Thomin A, Zacharopoulou C, Bazot M, et al. Role of protective defunctioning stoma in colorectal resection for endometriosis. J Minim Invasive Gynecol. juin 2014;21(3):472-9.

14. Gu W, Wu S. Meta-analysis of defunctioning stoma in low anterior resection with total mesorectal excision for rectal cancer: evidence based on thirteen studies. World J Surg Oncol. 24 janv 2015;13:9.

15. Montedori A, Cirocchi R, Farinella E, Sciannameo F, Abraha I. Covering ileo- or colostomy in anterior resection for rectal carcinoma. Cochrane Database Syst Rev. 12 mai 2010;(5):CD006878.

16. Ledu N, Rubod C, Piessen G, Roman H, Collinet P. Management of deep infiltrating endometriosis of the rectum: Is a systematic temporary stoma relevant? J Gynecol Obstet Hum Reprod. janv 2018;47(1):1-7.

17. Camilleri-Brennan J, Steele RJC. Prospective analysis of quality of life after reversal of a defunctioning loop ileostomy. Colorectal Dis Off J Assoc Coloproctology G B Irel. mai 2002;4(3):167-71.

18. Siassi M, Hohenberger W, Lösel F, Weiss M. Quality of life and patient's expectations after closure of a temporary stoma. Int J Colorectal Dis. déc 2008;23(12):1207-12.

19. Bonin E, Bridoux V, Chati R, Kermiche S, Coget J, Tuech JJ, et al. Diverting stomarelated complications following colorectal endometriosis surgery: a 163-patient cohort. Eur J Obstet Gynecol Reprod Biol. janv 2019;232:46-53.

20. Wiggins T, Markar SR, Arya S, Hanna GB. Anastomotic reinforcement with omentoplasty following gastrointestinal anastomosis: A systematic review and metaanalysis. Surg Oncol. sept 2015;24(3):181-6.

21. Vesale E., Boudy A. S., Zilbermann S., Bendifallah S., Ilenko A., Darai E. « Prévention de la fistule rectovaginale après résection colorectale enbloc et hystérectomie pour endométriose profonde ». Gynécologie Obstétrique Fertilité \& Sénologie [En ligne]. avril 2019. Vol. 47, n4, p. 378-380. Disponible sur : < https://doi.org/10.1016/j.gofs.2019.01.009 >

22. Boudy A-S, Vesale E, Arfi A, Owen C, Jayot A, Zilberman S, et al. Prevesical peritoneum interposition to prevent risk of rectovaginal fistula after en bloc colorectal resection with hysterectomy for endometriosis: Results of a pilot study. J Gynecol Obstet Hum Reprod. 21 nov 2019;101649. 


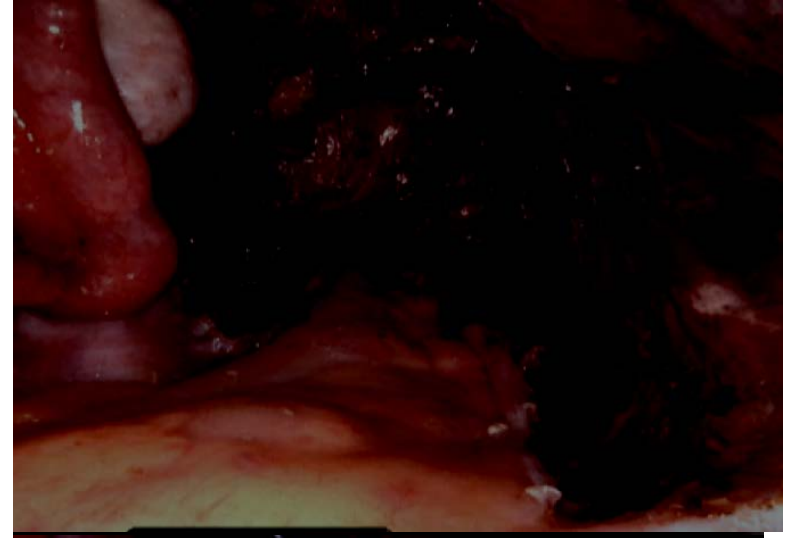

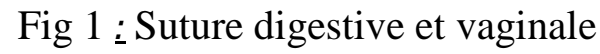




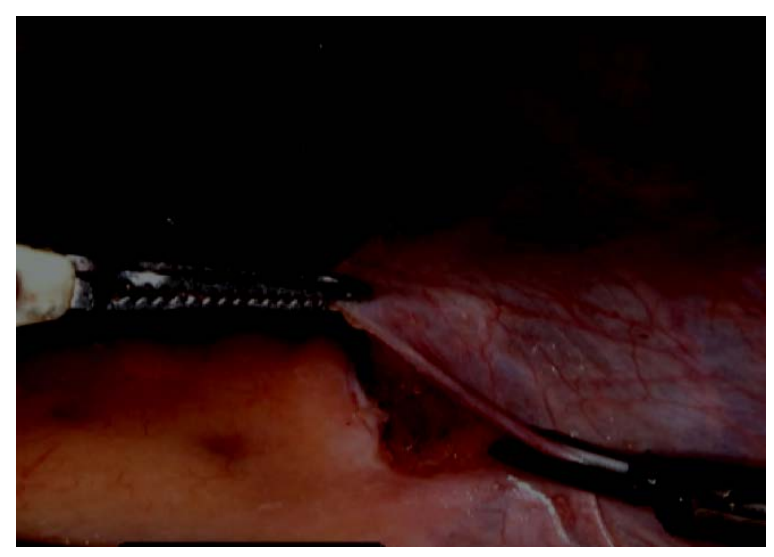

Fig 2: Découpe péritonéale en interne de l'uretère droit 


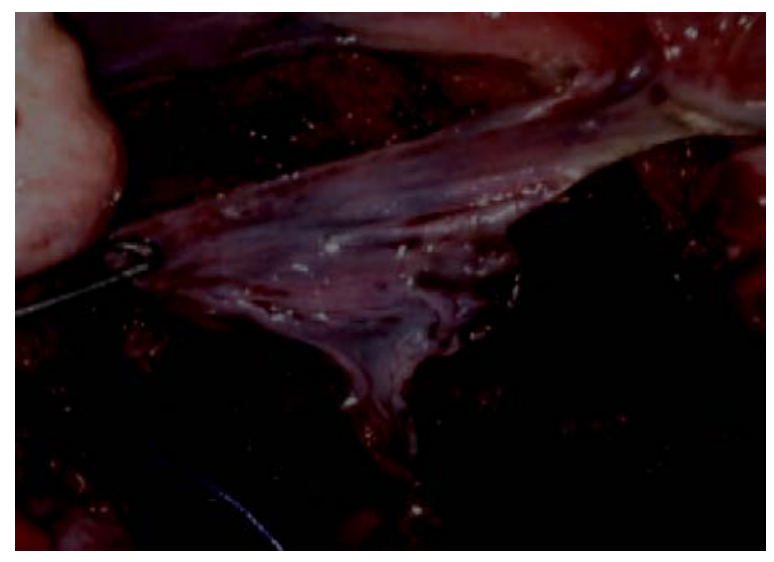

Fig 3: Plaie vaginale recouverte par la découpe péritonéale 


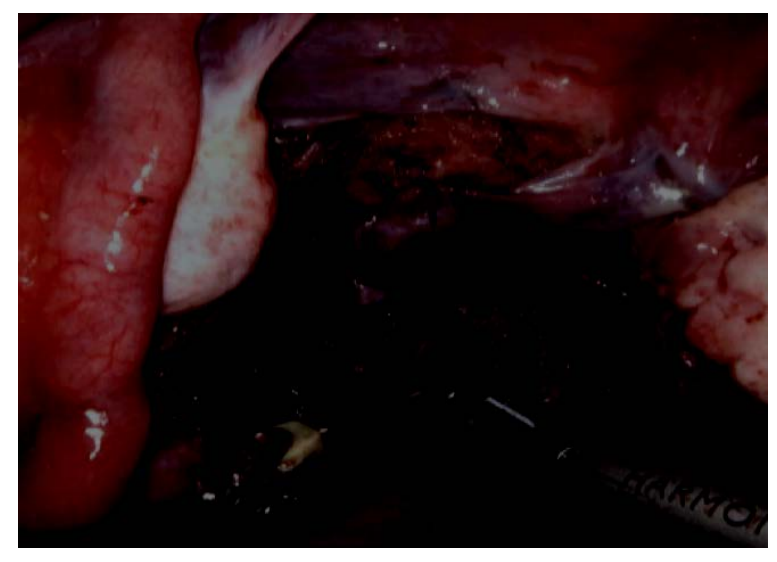

fig 4: découpe péritonéale fixée par des points séparés 